\title{
Two-Layer Network Equivalent for Electromagnetic Transients
}

\author{
Mohamed Abdel-Rahman, Member, IEEE, Adam Semlyen, Life Fellow, IEEE, and M. Reza Iravani, Fellow, IEEE
}

\begin{abstract}
The frequency spectrum of an external system used in the simulation of electromagnetic transients shows many peaks due to resonance effects of the leading transmission lines. Therefore, the two-level equivalent we developed contains simplified lines for the leading part of the system and lumped elements represented by rational functions as a correction for the rest of the external system. This leads to a computationally more efficient model, over a wide frequency range, than the existing equivalents with only lumped parameter components. The paper describes how the parameters of the new equivalent are optimized for most accurate fitting of the input admittance while assuring its positive realness and accuracy at dc and power frequency. Generators in the external system are represented in the equivalent as Norton current sources at the connecting terminals. The equivalents can be single or multiport.
\end{abstract}

Index Terms-External equivalents, multiport system, Norton equivalent, positive realness, quadratic programming, rational approximation, sequential quadratic programming.

\section{INTRODUCTION}

$\mathbf{F}$ OR the simulation of electromagnetic transients, a system can be divided into a study zone where the transient phenomena occur, and an external system often of very wide extension. Unlike the study zone, the external system can be represented as a linear system. There exists successful procedures for finding equivalents for the external system, which greatly reduce the computational burden of the simulations [1], [2]. Still, this remains a bottleneck in real-time simulations.

It has however been observed that the external system has a particular characteristic due to the fact that its most important part is transmission lines. In the frequency domain, even a single line produces a spectrum with numerous regularly placed peaks, so that its approximation by lumped parameter elements requires many $R, L, C, G$ resonant groups. It is then clear that if we keep some of the leading part of the original external network in the form of transmission lines-albeit simplified ones-then much of the burden of fitting the external system will be removed. We will only need a correction via some rational functions due to the simplified line representations and to account for the deeper layers of the system. We have thus a surface layer consisting of simple line models and a deep layer modeled by rational functions. This is the main underlying idea of the proposed two-layer equivalent. It is more efficient than traditional ones consisting of only lumped parameters.

Manuscript received January 15, 2003. This work was supported by the Natural Sciences and Engineering Research Council of Canada.

The authors are with the Department of Electrical and Computer Engineering, University of Toronto, ON M5S 3G4, Canada (e-mail: Mohammed@power.ele.utoronto.ca; adam.semlyen@utoronto.ca; iravani@ccf.utoronto.ca).

Digital Object Identifier 10.1109/TPWRD.2003.817749
There exist practical requirements for the external equivalent: it has to be accurate over a wide frequency range and in particular at power frequency and at $0 \mathrm{~Hz}$; it must also be positive-real, as it represents a passive system and must assure stable simulation. Our tools for achieving all of this are quadratic programming (QP) and sequential quadratic programming (SQP); the variables are increments to the initial parameters of the equivalent (i.e., of the leading simplified lines and the corrective rational functions).

The two-level equivalent described above can be extended for a multiport system. It can also represent accurately the effect of voltage sources in "active" networks by using a Norton current source at the connecting terminal(s). Thus, we obtain an active external equivalent. This is not a traditional Norton equivalent because the passive part of the system is represented by retaining transmission lines for the leading part of the external network.

In Section II of the paper, we present the basic underlying equations for the equivalent. In Section III, we describe the optimization procedure. Section IV deals with multiport equivalents. Section V describes the treatment of systems with active elements. Section VI represents the application of the equivalent to sample test cases. Section VII states the main conclusions.

\section{BASIC THEORY}

\section{A. Introducing the New Method}

An external system observed from a single or several ports may be partitioned into two regions: the outer part or surface region, and the inner part or deep region; see Fig. 1(a). The impact of the outer part and of the inner part on the input admittance varies with frequency. At low frequencies, both the inner and outer part contribute to the admittance. However, at higher frequencies, the role of the inner part drastically diminishes due to high attenuation. This means that the inner part can be approximated by a relatively smooth function, possibly a low-order rational function whose presence affects the input admittance mainly at the lower frequency range.

The previous argument leads to the idea that the system can be accurately and simply approximated by two layers corresponding to the "surface" region and the "deep" region; see Fig. 1(b). The first layer is a set of simplified transmission line representations that cover the whole frequency spectrum. The second is a rational transfer function that compensates for (i) the inaccuracy due to the simplified line models and (ii) the effect of the rest of the system. This provides a versatile tool to overcome problems of stability and positive realness, for, in case it was not possible to obtain a stable-positive-real approximation of the deep region, one could either increase the complexity of 

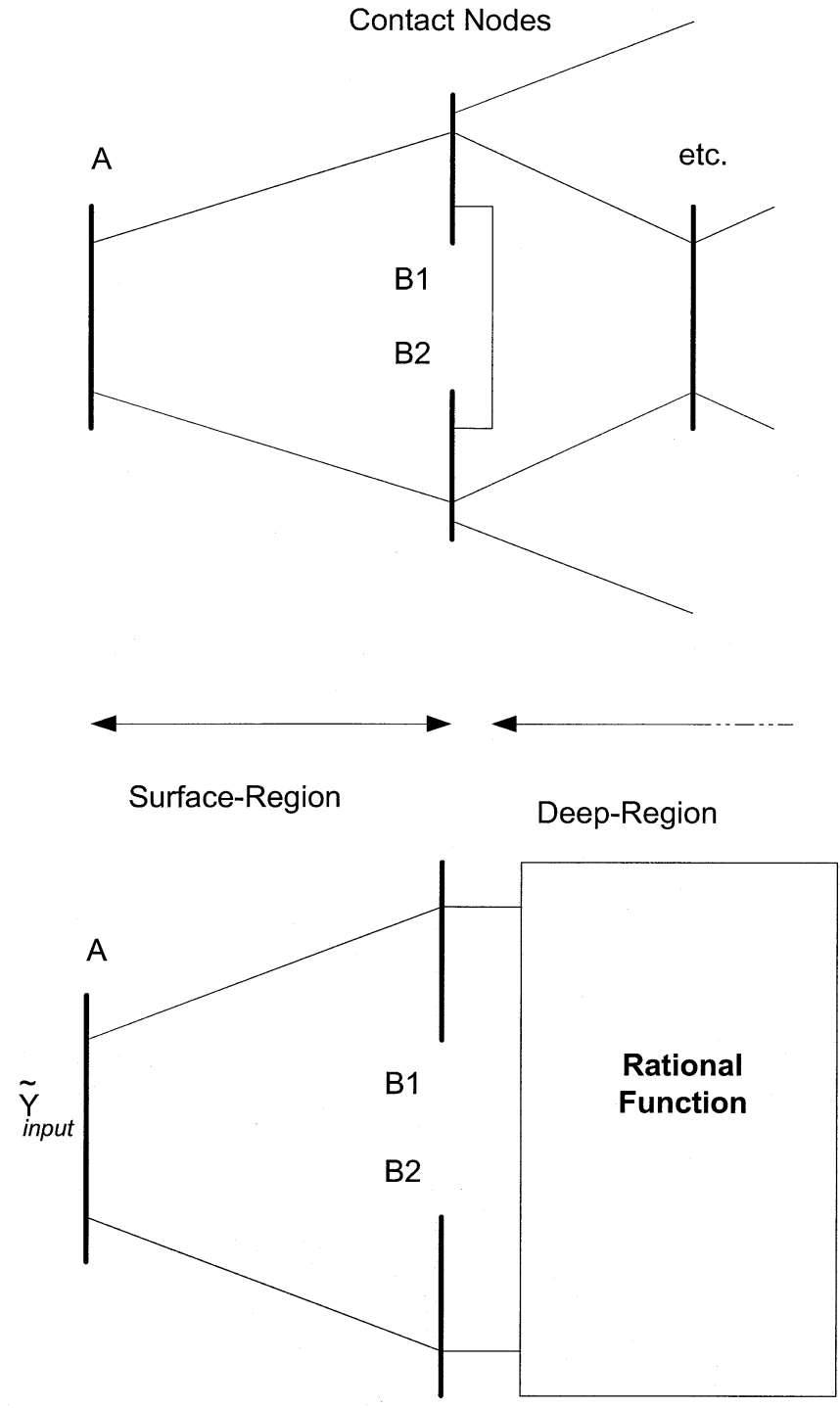

Fig. 1. (a) Partitioned representation of original network and (b) its equivalent.

the simplified transmission line models in the surface-layer or make this layer deeper.

The following subsections describe the models used for transmission lines and the rational function approximation for the inner part.

1) Transmission Line Models: A transmission line is characterized by a propagation function $H_{p}$ and a characteristic impedance $Z_{c}$. Both $H_{p}$ and $Z_{c}$ are frequency dependent functions. The propagation function $H_{p}$ defines [3] the relationship between the incident wave $V_{\text {incid }}$ and the reflected wave $V_{\text {refl }}$ by

$$
V_{\text {incid }}=H_{p} V_{\text {refl }}
$$

with

$$
H_{p}=H_{0} H_{s h}
$$

where $H_{o}=e^{-j \omega \tau} . \tau$ is the time delay along the line, and $H_{s h}$ is the shaping component. $H_{s h}$ can be approximated as a strictly proper rational function.
The rational approximation of the characteristic impedance is proper and can be written as the sum of partial fractions [4]

$$
k_{0}+\sum_{i}^{n} \frac{r_{i}}{s+p_{i}}
$$

where $k_{0}$ is a constant term, $p_{i}$ is the $i$ th pole, $r_{i}$ is the residue for the $i$ th fraction, and $s$ stands for $j \omega$.

2) Deep-Region Approximation: The deep-region is represented by a lumped parameter equivalent with emphasis on the low-frequency behavior. This lumped parameter equivalent must be stable. It generally takes the form of an $n \times n$ matrix, where $n$ is the number of the contact nodes between the surface-region and the deep-region; see Fig. 1. There are several approaches to find a rational function approximation to represent the deep-region. An efficient and accurate program to obtain it is vector fitting [5].

It is worthwhile at this point to state the steps of the proposed approach.

1) Partition the external network into a surface-region and a deep-region.

2) Find a simplified approximation for transmission lines in the surface region.

3) Find an equivalent for the deep-region as a low-order, lumped-parameter model.

4) Find the approximate input admittance $Y_{\text {input }}$ of Fig. 1(b) corresponding to the previously mentioned approximations, as developed in Section II-B below. These approximate parameters constitute a starting initial guess for the search for the optimal equivalent.

5) Optimize the equivalent, while imposing constraints for stability and positive realness. The optimization approach is developed in Section III.

\section{B. Input Admittance and Its Linearization}

The equivalent of Fig. 1(b) is composed of a layer of simplified transmission lines and a rational transfer function to account for the deep part of the network. The nodal equations of the transmission lines are

$$
\left[\begin{array}{c}
I_{A} \\
-I_{B}
\end{array}\right]=\left[\begin{array}{ll}
Y_{A A} & Y_{A B} \\
Y_{B A} & Y_{B B}
\end{array}\right]\left[\begin{array}{c}
V_{A} \\
V_{B}
\end{array}\right]
$$

where $I_{B}=\left[\begin{array}{ll}I_{B 1} & I_{B 2}\end{array}\right]^{T}$ is the vector of the injected currents from buses $B 1$ and $B 2$ into the deep-region, and $V_{B}=\left[\begin{array}{ll}V_{B 1} & V_{B 2}\end{array}\right]^{T}$ is the vector of bus voltages. Thus

$$
I_{B}=Y_{\text {deep }} V_{B}
$$

where $Y_{\text {deep }}$ is the rational function approximation of the input admittance matrix of the deep network. Substituting $I_{B}$ from (5) into (4), we get the input admittance as seen from terminal $A$

$$
Y_{\text {input }}=Y_{A A}-Y_{A B}\left(Y_{B B}+Y_{\text {deep }}\right)^{-1} Y_{B A} \text {. }
$$

Linearizing the expression for $Y_{\text {input }}$ gives

$$
Y_{\text {input }}=Y_{\text {input }}^{0}+J \Delta r
$$


where $Y_{\text {input }}^{0}$ is the initial approximation of the input admittance, $Y_{\text {input }}$ is the modified approximation, $J$ is the Jacobian, and $\Delta r$ is the change in the model parameters.

\section{OPTIMIZED FITTING}

To enhance the accuracy of the fitting of the equivalent, a search for an optimal $\Delta r$ of (7) is performed such that $Y_{\text {input }}$ of (7) approximates as closely as possible its calculated value for the original network of Fig. 1(a). First, we consider the lumped parameter component of the equivalent. The parameters that can be optimized are, as in (3), (i) the residues $r_{i}$ for the deep-region, with special care given to complex poles to keep them in conjugate pairs and (ii) the poles $p_{i}$ for the deep-region. To avoid stability problems, only the imaginary parts of the complex poles are optimized.

Optimizing the surface-region parameters can also enhance the accuracy of the equivalent. The first set of parameters to optimize is the set of delay times, $\tau$, of the transmission lines. The second set is the characteristic impedance parameters where again both poles and residues are available for optimization. Following the same steps as for the deep-region of the network, we optimize the residues and the imaginary parts of the poles. The only quantity that has been left out of the optimization is the shaping part of the propagation function because of the complexity of the calculations, but that could also be performed as an additional step to achieve higher accuracy.

To sum up, the initial approximation provides a crude starting point, from which optimization procedures are used to fine-tune the model to achieve better accuracy. Theoretically, all parameters of the model can be changed during optimization. However, in most cases, it is enough to modify the residues of the deepregion. If this does not provide the required accuracy, then the poles are also considered for optimization. The optimization of surface-region parameters is computationally more demanding. Thus, it is advisable to use them only as the last resort to obtain the desired accuracy.

\section{A. Calculation of Jacobian}

This subsection describes the calculation of the Jacobian in some detail for the deep-region parameters and briefly for the surface-region parameters. For the sake of simplicity, we will assume that the deep-region admittance is a scalar.

1) Jacobian for Deep Network Parameters: The rational function that represents the deep region is

$$
Y_{\text {deep }}=k_{0}+\sum_{i=1}^{n} \frac{r_{i}}{s+p_{i}}+\sum_{i=n+1}^{n+\frac{m}{2}}\left\{\frac{r_{i}}{s+p_{i}}+\frac{r_{i}^{*}}{s+p_{i}^{*}}\right\}
$$

where $n$ and $m$ are the number of real and complex poles, respectively.

The derivative of (6) with respect to a real parameter $\rho$ is

$\frac{\partial Y_{\text {input }}}{\partial \rho}=Y_{A B}\left(Y_{B B}+Y_{\text {deep }}\right)^{-1} \frac{\partial Y_{\text {deep }}}{\partial \rho}\left(Y_{B B}+Y_{\text {deep }}\right)^{-1} Y_{B A}$

where $\rho$ is the variable considered for optimization. The calculations are shown in some detail in Appendix A.
2) Jacobian for the Surface Region: The surface-region is a set of simplified transmission lines. The first element that should be optimized is the delay time $\tau$. Obtaining the first derivative with respect to $\tau$ is a straightforward process. The other parameters that may be optimized are those of the characteristic admittance; namely, the residues and the imaginary parts of the conjugate poles. (See Appendix B).

\section{B. Unconstrained Least Squares Error Minimization}

Given the calculated Jacobian matrix and replacing in the left-hand side of (7) the actual input admittance, the only unknown is the vector $\Delta r$

$$
Y_{\text {input }}-Y_{\text {input }}^{0}=J \Delta r
$$

Equation (10) represents a set of over-determined linear equations. Its solution should be obtained in a least squares sense. The problem is eventually reduced to a minimization problem of the form

$$
\text { minimize : } \quad \sum\left\|\left(Y_{\text {input }}-Y_{\text {input }}^{0}\right)-J \Delta r\right\|_{2}^{2} \text {. }
$$

As all of the elements of (11) but $\Delta r$ are complex quantities, a slight modification is necessary to break the problem into its real and imaginary parts. This is needed to use available optimization techniques.

\section{Constrained Optimization}

The previous subsection showed how to minimize the error between the equivalent and the exact input admittance. However, there is no guarantee that either the deep-region or the approximated equivalent will not violate the positive-realness criterion for admittance matrices. Hence, to ensure positiverealness, appropriate constraints should be imposed.

First, consider the positive-realness of the input. It can be seen, based on (7), that the constraint of positive-realness for the input admittance to the system is

$$
-\Re e\left\{Y_{\text {input }}^{0}\right\} \geq \Re e\{J \Delta r\} .
$$

Thus, the solution of (11) is a constrained optimization problem that can be solved by quadratic programming $(\mathrm{QP})$.

The previous discussion is solely concerned with the fulfillment of positive-realness of the driving-point admittance. However, one part of the equivalent is the lumped parameter part, which should also, by itself, be positive-real. A sufficient condition of positive-realness for the deep-region is

$$
\text { Eigenvalues }\left\{\Re e\left(Y_{\text {deep }}^{o}\right)\right\} \geq 0 \text {. }
$$

By imposing (13), positive-realness of the deep-region is guaranteed. This requires, due to nonlinearities, a shift from QP to sequential quadratic programming (SQP). We have used MATLAB routines for both QP and SQP.

\section{Multiport EQuivalents}

Fig. 2 shows a network in which the study zone is interfaced with the external system through two ports. In general, the interface can be a multiport. This section only concentrates on the two-port equivalent. 


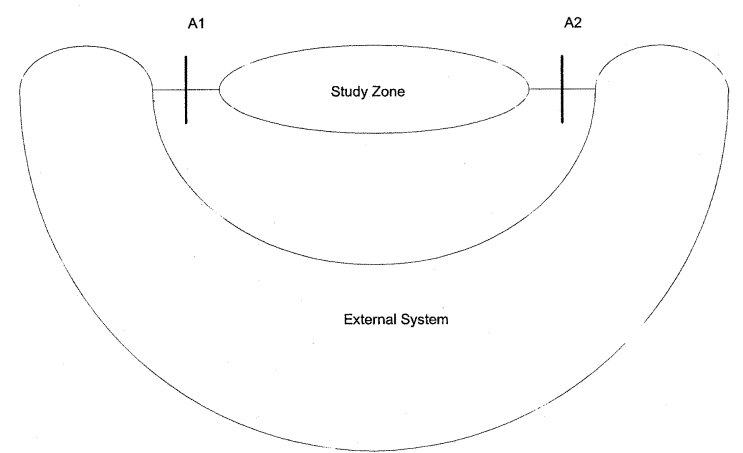

Fig. 2. Study zone and the two-port external network.

\section{A. Unconstrained Least Squares Optimization}

Consider a single-phase, two-port network as the external network (Fig. 2). The input admittance $Y_{\text {input }}$ is a $2 \times 2$ matrix. The external network is composed of a surface-region, that is represented by a number of low-order transmission lines, and a deep-region which has lumped-parameter representation. The specified and the calculated admittances of the external system are

$$
Y_{\text {input }}=\left[\begin{array}{ll}
y_{11} & y_{12} \\
y_{21} & y_{22}
\end{array}\right], \quad \widetilde{Y}_{\text {input }}=\left[\begin{array}{ll}
\widetilde{y}_{11} & \widetilde{y}_{12} \\
\widetilde{y}_{21} & \widetilde{y}_{22}
\end{array}\right] .
$$

The objective function to be minimized is

$$
w=\left\|Y_{\text {input }}-\tilde{Y}_{\text {input }}\right\|_{F}^{2}
$$

or

$$
w=\sum\left|y_{i j}-\widetilde{y}_{i j}\right|^{2} \quad(i, j=1,2) .
$$

In addition, (7) should be rewritten to represent four sets of equations, one corresponding to each element in (16) corresponding to the matrices (14)

$$
y_{i j}-\widetilde{y}_{i j}=\left(\sum_{k=1}^{n} \frac{\partial Y_{\text {input }}}{\partial \rho_{k}} \cdot \Delta \rho_{k}\right)_{i j} \quad(i, j=1,2) .
$$

This formulation reduces the problem complexity to that of (7), and thus, the same method of solution is applicable.

Generalization of this approach to an $n$-port system is conceptually straightforward. Moreover, this approach can be expanded to an $m$-phase system without significant alteration, provided that the system is transformed into modal domain where it can be treated as $m$ decoupled single-phase networks. Each of these networks can be treated independently as discussed before.

\section{B. Constrained Optimization}

The previous subsection disregarded the fact that the input admittance matrix should not violate the positive-realness criterion. The constraint is similar to that of (13) and is given as

$$
\text { Eigenvalues }\left\{\Re e\left(Y_{\text {input }}\right)\right\} \geq 0 \text {. }
$$

SQP is one method to impose (18) as the optimization constraint.
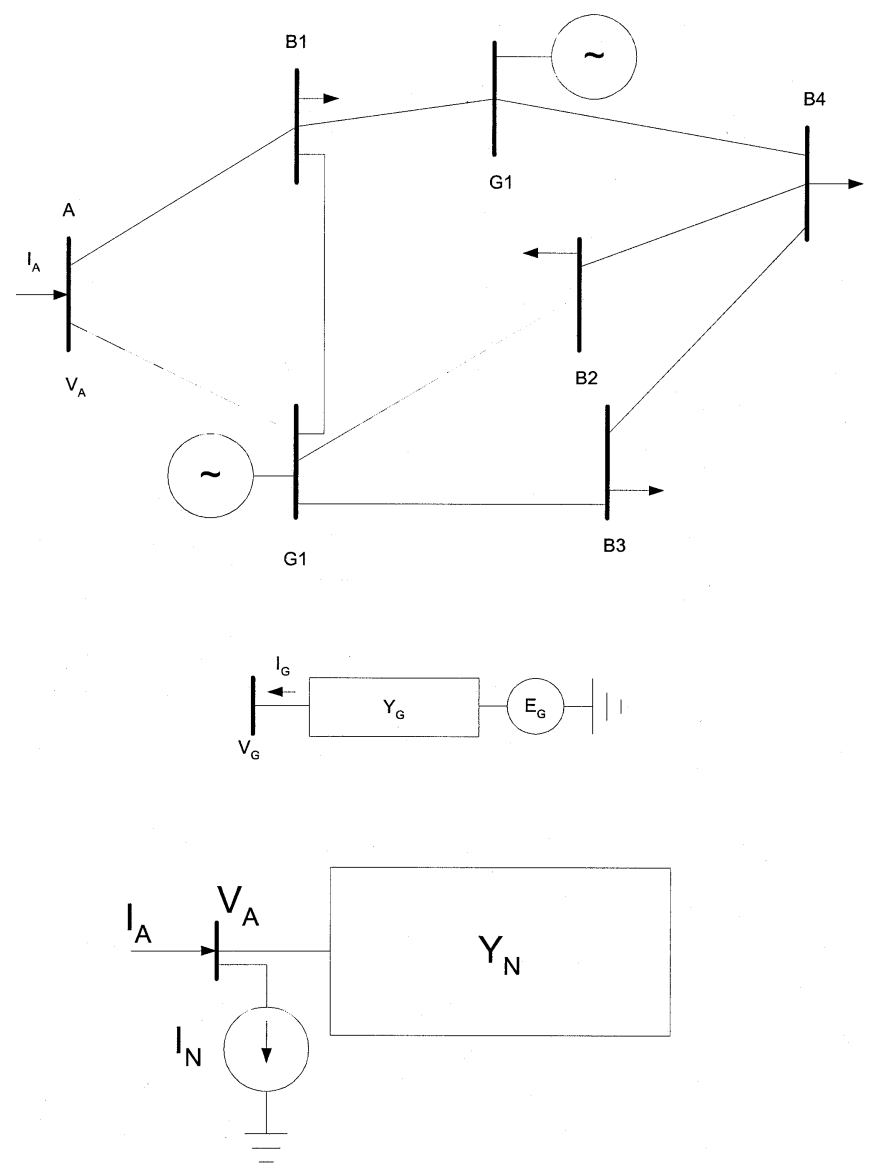

Fig. 3. (a) Original system with generators. (b) Generator bus in detail. (c) Equivalent network.

\section{SySTEMS With GENERATORS}

In this section, we extend the discussion to cover external networks that contain generators or other active sources. As the external system is assumed to be linear, an equivalent of the active system in the form of a (Norton) current source and a passive network is possible. The following subsection shows the development of the equivalent.

\section{A. Active External Equivalent}

Consider the external system of Fig. 3. The system generally has connection buses (A), load buses (B), and generator buses (G). The nodal equations are

$$
\left[\begin{array}{c}
I_{A} \\
I_{G} \\
I_{B}=0
\end{array}\right]=\left[\begin{array}{ccc}
Y_{A A} & Y_{A G} & Y_{A B} \\
Y_{G A} & Y_{G G} & Y_{G B} \\
Y_{B A} & Y_{B G} & Y_{B B}
\end{array}\right]\left[\begin{array}{c}
V_{A} \\
V_{G} \\
V_{B}
\end{array}\right] .
$$

After a reduction process shown in Appendix $C$, we obtain

$$
I_{A}=Y_{N} V_{A}+I_{N}
$$

where $Y_{N}$ and $I_{N}$ are the Norton admittance and current, respectively, given in (36) and (37) in Appendix C. $Y_{N}$ is the admittance of the passive system. Any inaccuracy in the Norton equivalent is due to the approximations in the computation of $Y_{N}$ [equal to $Y_{\text {input }}$ of (6)], since the calculation of $I_{N}$ does not involve approximations. 


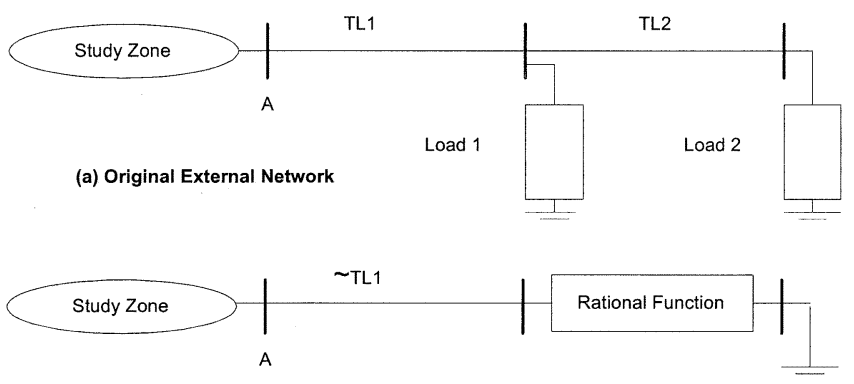

(b) Equivalent

Fig. 4. (a) Radial test system of example 1 and (b) equivalent.
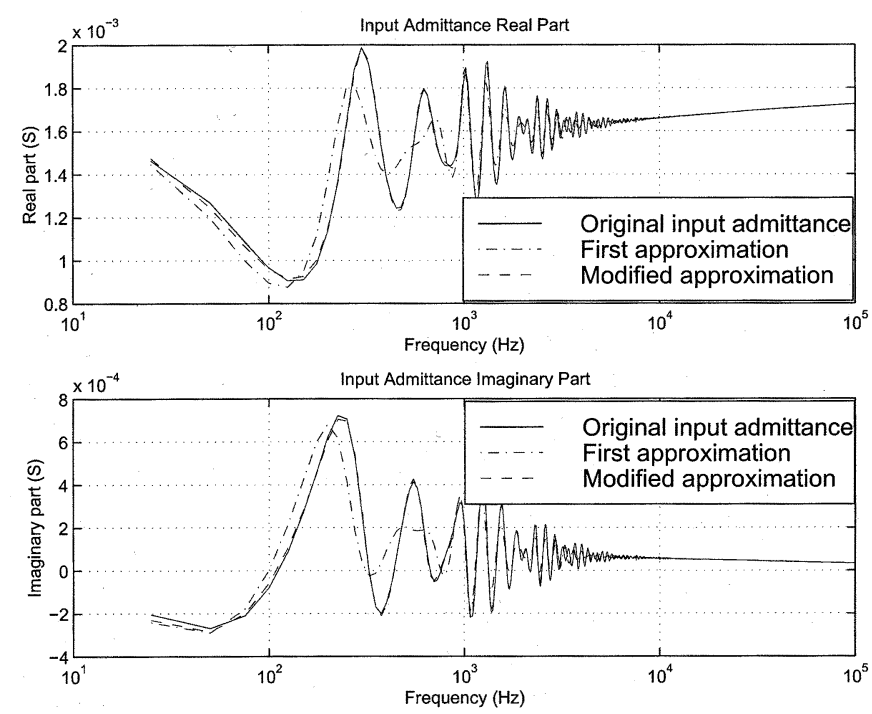

Fig. 5. Input admittance, example 1.

In conclusion, the procedure is

1) Obtain an equivalent for the passive system (i.e., for the system where all generator voltages have been set to zero as was described in Sections II and III).

2) Calculate $I_{N}$ from (38) at the power frequency only. In this way, the effect of the generators is taken into account.

\section{B. Multiport Networks and Harmonic Sources}

Equation (20) is also applicable to multiport systems. The linearity of the external system makes it possible to obtain Norton current sources at different frequencies, if harmonic sources are to be considered.

\section{COMPUTATIONAL Results}

This section provides three examples to illustrate the feasibility of the new method. In the first example, modification of the deep network's residues is enough to produce adequate correction to the result. The second example shows that for an interconnected network, the situation will be simpler, as the error between various transmission-line approximations acts such that the discrepancy between the original surface-region and the approximation is less than in the case of a single transmission line. Thus, the role of the deep network further diminishes. The third example explores the potential of the new technique applied to an active system.
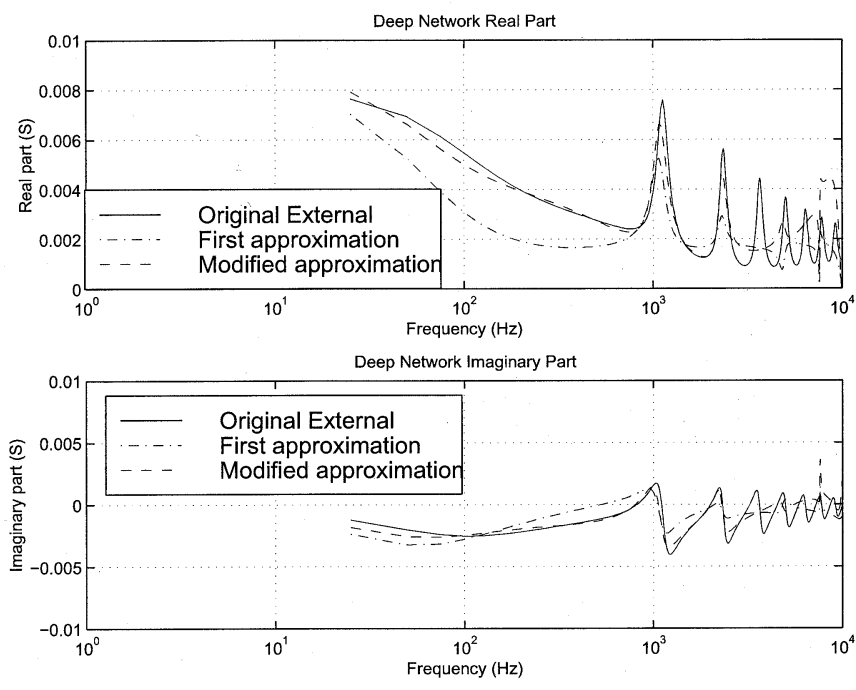

Fig. 6. Deep-region, example 1.

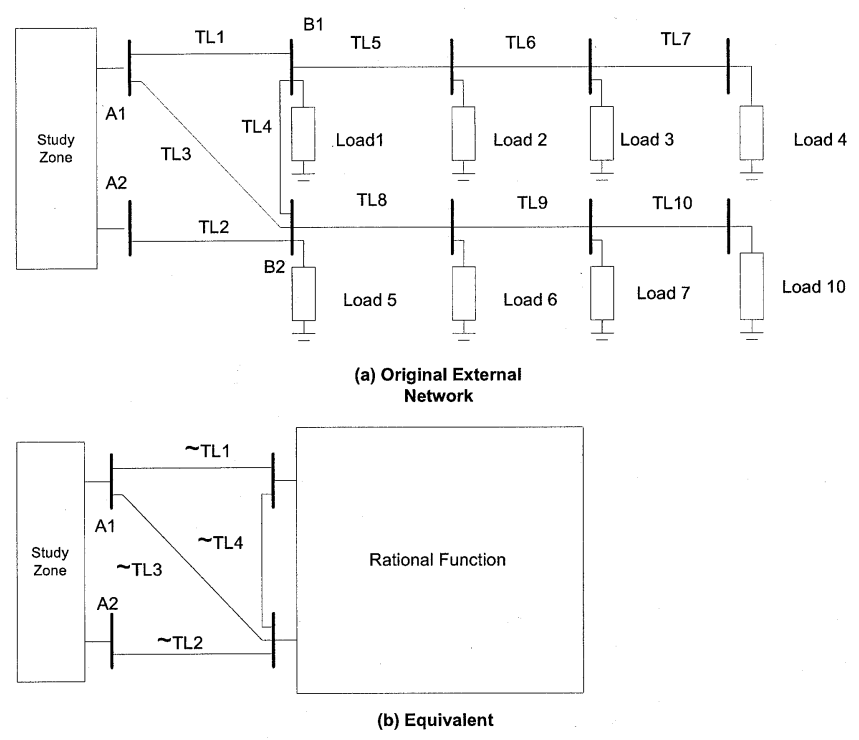

Fig. 7. Test system for example 2 .

\section{A. Examples}

Example 1: Fig. 4(a) shows a radial, single-phase test system composed of two transmission lines, of $400-\mathrm{km}$ and $100-\mathrm{km}$ length [6]. The two loads are RL branches. The leading line is represented by an approximated shaping factor and characteristic impedance of sixth-order. The deep network is represented by a rational function of 15 th-order. The equivalent is schematically shown in Fig. 4(b). Fig. 5 shows the input admittance and its approximations, from an initial to an improved model obtained by parameter optimization. The correction from the first approximation to the final one is obvious. Ten iterations were enough to achieve this accuracy. Fig. 6 shows the original admittance of the deep network, the first approximation, and the optimized one. Appendix D contains the parameters of this example.

Example 2: Fig. 7(a) shows a two-port single-phase test system composed of ten interconnected transmission lines [6]. The equivalent consists of a surface layer of approximated 

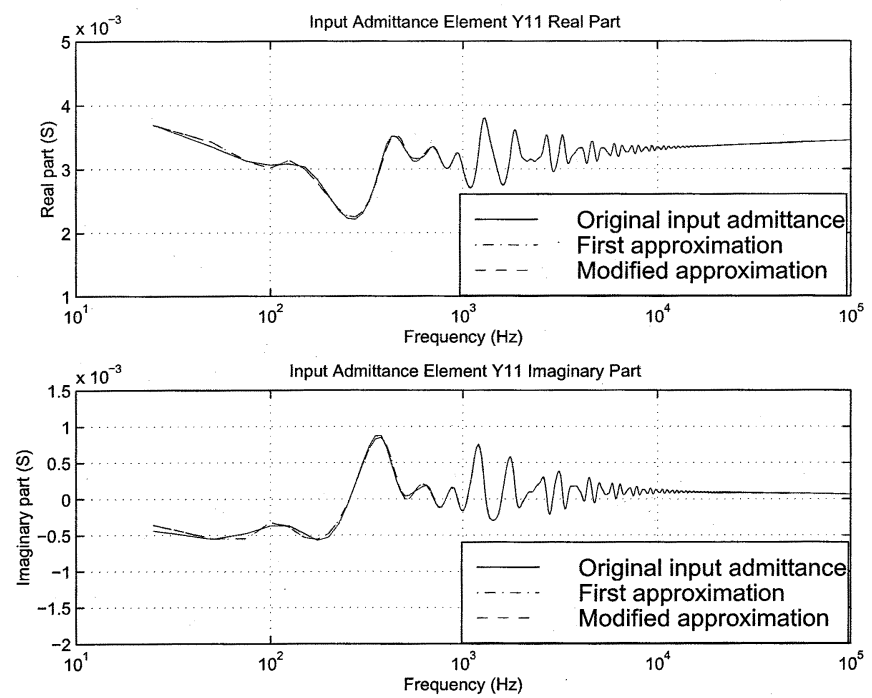

Fig. 8. Representation of element $y_{11}$ of input admittance matrix, example 2.
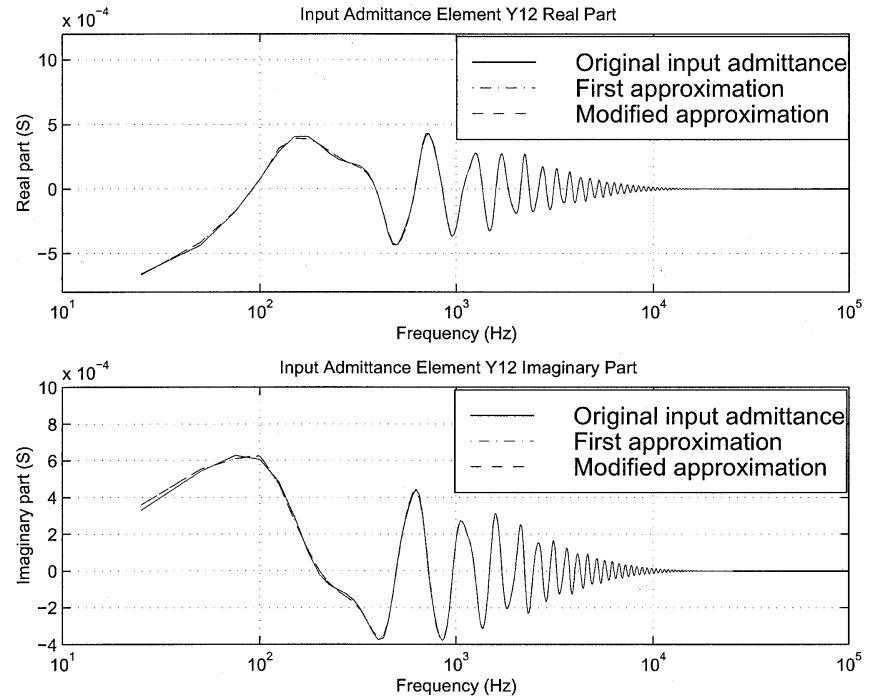

Fig. 9. Representation of element $y_{12}$ of input admittance matrix, example 2.

transmission lines and a deep region. The deep-region is represented by a diagonal matrix, due to the absence of coupling between connection nodes within the deep region. Transmission line approximations are similar to the previous example. The deep region is approximated by a second-order matrix rational function.

The surface region is composed of four transmission line simplified models. The equivalent is schematically shown in Fig. 7(b). Figs. 8, 9, and 10 show the elements of the input admittance matrix. We note that due to the interconnections, the first approximation itself is already close to the original characteristic. The merits of this approach are more striking when compared with the conventional approach where hundreds of poles need to be used to obtain the same accuracy. Another advantage is the reduced simulation time with the low-order functions used in the new model compared with the simulation time with a conventional approach. Problems of
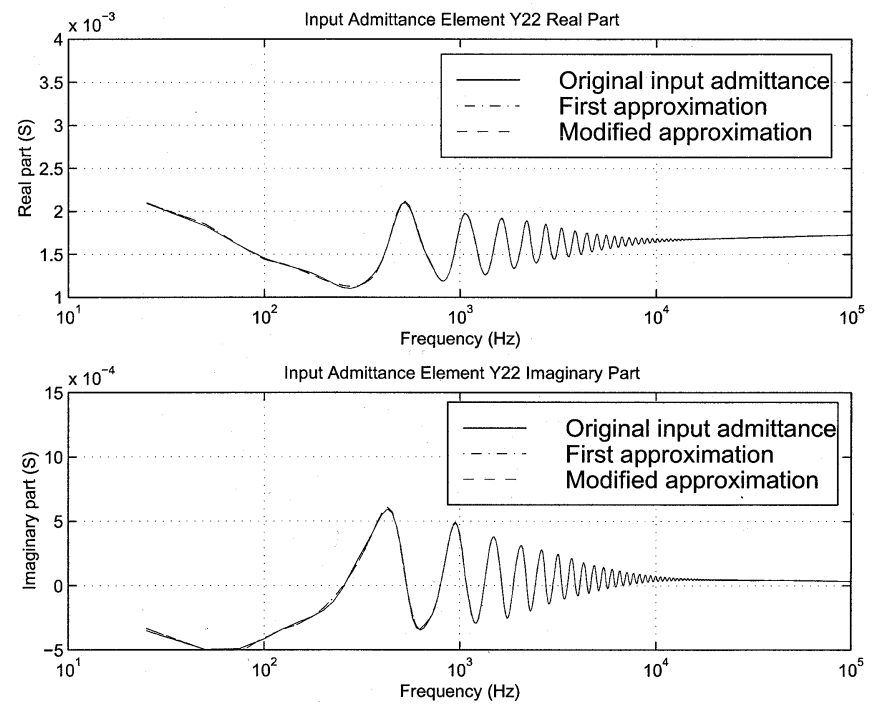

Fig. 10. Representation of element $y_{22}$ of input admittance matrix, example

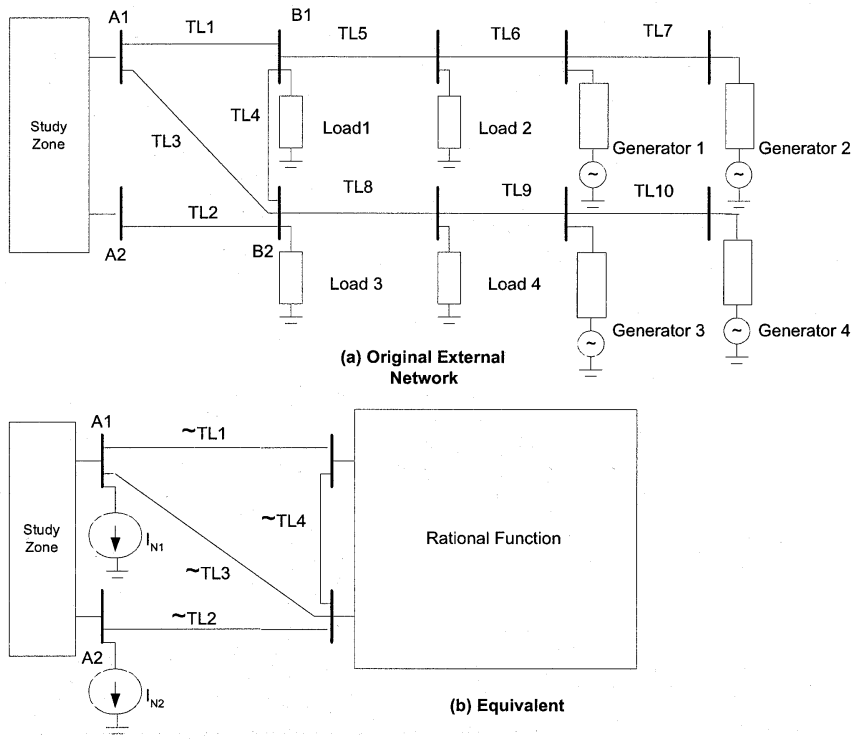

Fig. 11. (a) Original system. (b) The equivalent.

positive-realness and stability are inherently treated in the new method.

Example 3: Fig. 11(a) shows a single-phase system that contains four generators operating at $60 \mathrm{~Hz}, 10 \mathrm{kV}$. The equivalent is schematically shown in Fig. 11(b). The Norton current source is a set of current sources as shown in Fig. 11(b). Terminal A1 is short-circuited at $0.16 \mathrm{~s}$ and terminal A2 at $0.1 \mathrm{~s}$. The results of the simulation of these switching events, using both the original network and the new equivalent are shown in Figs. 12(a) and 12(b). The responses are practically indistinguishable.

\section{B. Efficiency}

The efficiency of the two-layer network equivalent depends on both the system for which it is used and its practical implementation. For the cases examined above, we have found 

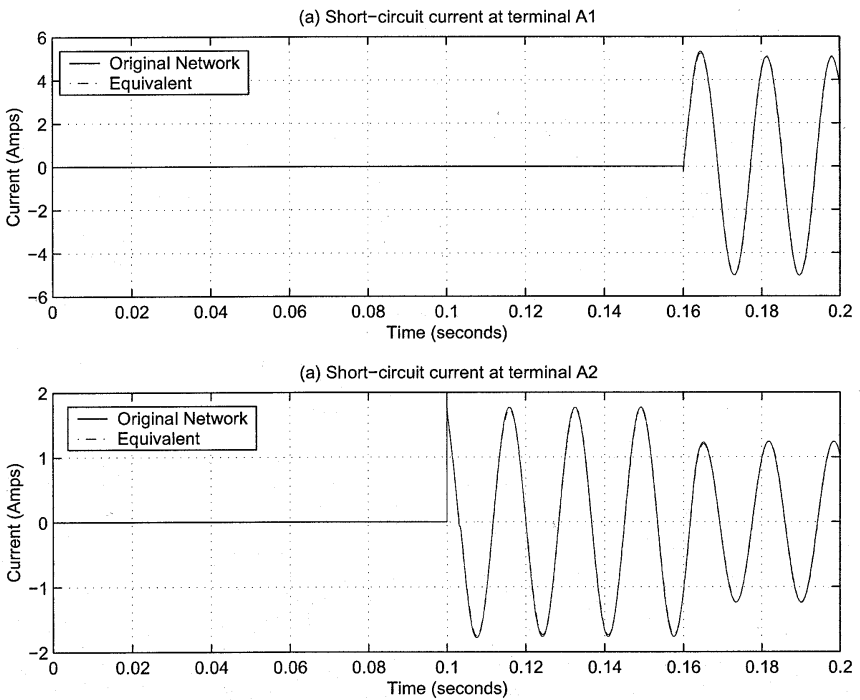

Fig. 12. (a) Short-circuit current at terminal A1. (b) Short-circuit current at terminal A2.

that the computational effort in terms of Mflops for the simulation of transients using the new equivalent is about eight times lower than using the original external system. For systems of realistic size, the results are even more in favor of the new equivalent [6]. Appendix E contains the parameters of example 2 and example 3.

\section{CONCLUSIONS}

A new methodology for obtaining an equivalent for an external network has been described. It includes some leading simplified line models for more efficient modeling of the external system. Optimization techniques have been used to achieve enhanced accuracy while satisfying the necessary conditions for a stable equivalent both in frequency and time domain. A salient feature of the proposed approach is that the instability problem can be always overcome by including more lines or by slightly increasing the complexity of the line models. The new equivalent is stable, positive-real, accurate, and fast to produce. Huge reduction in the computation time makes it an efficient tool for online calculations. The paper also provides a methodology for obtaining an equivalent of an active system. Examples are presented to show the effectiveness of the new method.

\section{APPENDIX A}

\section{DERIVATIVES}

In this appendix, the calculation of the derivatives of the deep network with respect to its parameters is described.

For instance, for a real residue the optimized parameter is

$$
\rho=r_{i} .
$$

The situation is slightly different for the case of complex residues as they come in conjugate pairs

$$
r_{i}=\alpha_{i}+j \beta_{i}, \quad r_{i}^{*}=\alpha_{i}-j \beta_{i} .
$$

Thus, for complex residues, the optimized parameters are $\alpha_{i}$ and $\beta_{i}$ independently rather than the residues $r_{i}$ and $r_{i}^{*}$. Therefore, for $\rho=\alpha_{i}$

$$
\frac{\partial Y_{\text {deep }}}{\partial \rho}=\frac{\partial Y_{\text {deep }}}{\partial r_{i}}+\frac{\partial Y_{\text {deep }}}{\partial r_{i}^{*}}
$$

and for $\rho=\beta_{i}$ :

$$
\frac{\partial Y_{\text {deep }}}{\partial \rho}=j\left(\frac{\partial Y_{\text {deep }}}{\partial r_{i}}-\frac{\partial Y_{\text {deep }}}{\partial r_{i}^{*}}\right) .
$$

The other variables that are related to the deep lumped parameter network are the complex parts $\delta$ of complex poles, for which

$$
\frac{\partial Y_{\text {deep }}}{\partial \rho}=j\left(\frac{\partial Y_{\text {deep }}}{\partial p_{i}}-\frac{\partial Y_{\text {deep }}}{\partial p_{i}^{*}}\right) \text {. }
$$

\section{APPENDIX B}

\section{JACOBIAN FOR SURFACE LAYER}

The Jacobian calculations with respect to surface-region parameters are more demanding than for deep-region parameters. The elements of the nodal admittance matrix of a distributed parameter transmission line model are given by

$$
\begin{aligned}
& Y_{A A}=\frac{1}{Z_{c}} \operatorname{coth}(\gamma \ell), \quad Y_{B B}=Y_{A A} \\
& Y_{A B}=-\frac{1}{Z_{c}} \operatorname{csch}(\gamma \ell), \quad Y_{B A}=Y_{A B}
\end{aligned}
$$

where $\gamma$ is the propagation constant and $\ell$ is the transmission line length. We rewrite the propagation function as

$$
H(s)=H_{s h} \cdot e^{-s \tau}
$$

where

$$
H_{s h}=\sum_{i=1}^{n} \frac{r_{i}}{s+p_{i}} .
$$

Given that

$$
\begin{aligned}
\operatorname{coth}(\gamma \ell) & =\frac{H+H^{-1}}{H-H^{-1}} \\
\operatorname{csch}(\gamma \ell) & =\frac{2}{H-H^{-1}}
\end{aligned}
$$

the derivative can be obtained with respect to any desired parameter.

\section{APPENDIX C}

\section{DETAILS FOR THE NORTON EQUIVALENT}

From the third row of (19)

$$
V_{B}=-Y_{B B}^{-1}\left(Y_{B A} V_{A}+Y_{B G} V_{G}\right) .
$$

By substituting (29) into (19), we get

$$
\begin{aligned}
& I_{A}=Y_{A A}^{\prime} V_{A}+Y_{A G}^{\prime} V_{G} \\
& I_{G}=Y_{G A}^{\prime} V_{A}+Y_{G G}^{\prime} V_{G}
\end{aligned}
$$


where

$$
\begin{aligned}
& Y_{A A}^{\prime}=Y_{A A}-Y_{A B} Y_{B B}^{-1} Y_{B A} \\
& Y_{A G}^{\prime}=Y_{A G}-Y_{A B} Y_{B B}^{-1} Y_{B G} \\
& Y_{G A}^{\prime}=Y_{G A}-Y_{G B} Y_{B B}^{-1} Y_{B A} \\
& Y_{G G}^{\prime}=Y_{G G}-Y_{A B} Y_{B B}^{-1} Y_{B G}
\end{aligned}
$$

or, in a matrix form

$$
\left[\begin{array}{c}
I_{A} \\
I_{G}
\end{array}\right]=\left[\begin{array}{cc}
Y_{A A}^{\prime} & Y_{A G}^{\prime} \\
Y_{G A}^{\prime} & Y_{G G}^{\prime}
\end{array}\right]\left[\begin{array}{c}
V_{A} \\
V_{G}
\end{array}\right]
$$

Also, from Fig. 3(b)

$$
I_{G}=Y_{G}\left(E_{G}-V_{G}\right)
$$

Substituting from (33) into (32), we obtain

$$
\begin{aligned}
{\left[\begin{array}{c}
I_{A} \\
Y_{G} E_{G}
\end{array}\right] } & =\left[\begin{array}{cc}
Y_{A A}^{\prime} & Y_{A G}^{\prime} \\
Y_{G A}^{\prime} & Y_{G G}^{\prime}+Y_{G}
\end{array}\right]\left[\begin{array}{c}
V_{A} \\
V_{G}
\end{array}\right] \\
V_{G} & =\left(Y_{G G}^{\prime}+Y_{G}\right)^{-1}\left(Y_{G} E_{G}-Y_{G A}^{\prime} V_{A}\right) .
\end{aligned}
$$

From (34) and (35)

$$
I_{A}=Y_{N} V_{A}+I_{N}
$$

where

$$
\begin{aligned}
Y_{N} & =Y_{A A}^{\prime}-Y_{A G}^{\prime}\left(Y_{G G}^{\prime}+Y_{G}\right)^{-1} Y_{G A}^{\prime} \\
I_{N} & =Y_{A G}^{\prime}\left(Y_{G G}^{\prime}+Y_{G}\right)^{-1} Y_{G} E_{G}
\end{aligned}
$$

\section{APPENDIX D}

\section{SYSTEM PARAMETERS (FIG. 4)}

The lines are 400- and 100-km, respectively. Loads 1 and 2 are lumped RL branches of 350-ohm, 50- $\mathrm{mH}$ and 50-ohm, $50-\mathrm{mH}$, respectively. Poles of the line propagation function are $-0.014 \mathrm{E} 04,-0.2145 \mathrm{E} 4,-1.2485 \mathrm{E} 4,-3.9731 \mathrm{E} 4$, $-4.3525 \mathrm{E} 4+\mathrm{j} 4.8893 \mathrm{E} 4$, and $-4.3525 \mathrm{E} 4-\mathrm{j} 4.8893 \mathrm{E} 4$ and the corresponding residues are $0.0029 \mathrm{E} 4,0.0368 \mathrm{E} 4,1.2558 \mathrm{E} 4$, $-2.2511 \mathrm{E} 4,0.4766 \mathrm{E} 4+\mathrm{j} 0.2957 \mathrm{E} 4$, and $0.4766-\mathrm{j} 0.2957 \mathrm{E} 4$, respectively. Poles of the line characteristic impedance are $-5.9328 \mathrm{E} 5,-0.8979 \mathrm{E} 5,-0.1366 \mathrm{E} 5,-0.0124 \mathrm{E} 5,-0.018 \mathrm{E} 5$, and $-0.0002 \mathrm{E} 5$, and the corresponding residues are $8.7048 \mathrm{E} 6$, 1.9319E6, 0.3683E6, 1.0662E6, 0.0971E6, and 0.0737E6, respectively. The constant term of the line characteristic impedance is 572.073-ohm.

\section{APPENDIX E}

\section{SySTEM PARAMETERS (FIG. 7 AND FIG. 11)}

The line lengths in kilometers are TL1 $=210$, TL2 $=300$, TL3 $=450$, TL4 $=250$, TL5 $=400$, TL6 $=100$, TL7 $=60$, TL8 $=400$, TL9 $=100$, TL10 $=60$. Load resistance $(\mathrm{ohm})$ and inductance $(\mathrm{mH})$ are Load 1: 350 and 50; Load 2: 50 and 50; Load 3: 50 and 50; Load 4: 50 and 50; Load 5: 350 and 50; Load 6: 50 and 50; Load 7: 50 and 50, and Load 8: 50 and 50. Resistance and inductance of each generator (Fig. 11) are $50 \Omega$ and $50 \mathrm{mH}$. The e.m.f. of each generator is $10-\mathrm{kV}$ peak value. $\mathrm{I}_{\mathrm{N} 1}$ and $\mathrm{I}_{\mathrm{N} 2}$ are $4.882 \mathrm{~A}$ peak at angle $2.486^{\circ}$ and 1.1827 -A peak at angle $2.402^{\circ}$, respectively.

\section{REFERENCES}

[1] A. S. Morched and V. Brandwajn, "Transmission network equivalents for electromagnetic transients studies," IEEE Trans. Power App. Syst., vol. PAS-102, pp. 2984-2994, Sept. 1983.

[2] A. S. Morched, J. H. Ottevangers, and L. Marti, "Multi-port frequency dependent network equivalent for EMTP," IEEE Trans. Power Delivery, vol. 8, pp. 1402-1412, July 1993.

[3] A. Semlyen and M. R. Iravani, "Frequency domain modeling of external systems in an electromagnetic transients program," IEEE Trans. Power Syst., vol. 8, pp. 527-533, May 1993.

[4] J. R. Marti, "Accurate modeling of frequency-dependent transmission lines in electromagnetic transient simulations," IEEE Trans. Power App. Syst., vol. 101, pp. 147-155, Jan. 1982.

[5] B. Gusatvsen and A. Semlyen, "Rational approximation of frequency domain responses by vector fitting," IEEE Trans. Power Delivery, vol. 14 , pp. 1052-1061, July 1999.

[6] M. Abdel-Rahman, "Frequency dependent hybrid equivalents of large networks," Ph.D. thesis, Univ. Toronto, 2001.

Mohamed Abdel-Rahman (M'00) received the B.Sc. degree in electrical engineering from Ain-Shams University in 1995, and the M.A.Sc. and Ph.D. degrees in electrical engineering from the University of Toronto, ON, Canada, in 1998.

Currently, he is an Assistant Professor at Ain Shams University.

Adam Semlyen (LF'97) was born in 1923 in Rumania. He received the Dipl. Ing. degree from the Polytechnic Institute of Timisoara, Rumania, and the Ph.D. degrees from Isai, Rumania.

He held academic positions at the Polytechnic Institute of Timisoara. In 1969, he joined the University of Toronto, Toronto, ON, Canada, where he is a Professor in the Department of Electrical and Computer Engineering, emeritus since 1988. His research interests include steady state and dynamic analysis as well as computation of electromagnetic transients in power systems.

M. Reza Iravani (M'85-SM'00-F'03) received the B.Sc. degree in electrical engineering from Tehran Polytechnic University, Iran, in 1976, and the M.Sc. and Ph.D. degrees in electrical engineering from the University of Manitoba, Winnipeg, Canada, in 1981 and 1985, respectively.

Currently, he is a Professor at the University of Toronto, ON, Canada. His research interests include power electronics and power system dynamics and control. 